\title{
РОЛЬ РЕГУЛЯЦИИ ГЕНОВ АПФ2/ТМРRSS2 ИЗОФОРМАМИ МИКРОРНК КИШЕЧНИКА В ПАТОГЕНЕЗЕ COVID-19
}

\author{
С. А. Нерсисян ${ }^{1,2}$ М. М. Ю. Шкурников ${ }^{3}$, А. И. Осипьянц ${ }^{3,4}$, В. И. Вечорко
}

${ }^{1}$ Национальный исследовательский университет «Высшая школа экономики», Москва, Россия

${ }^{2}$ Московский государственный университет имени М. В. Ломоносова, Москва, Россия

${ }^{3}$ Московский научно-исследовательский онкологический институт имени П. А. Герцена - филиал Национального медицинского исследовательского центра радиологии, Москва, Россия

${ }^{4}$ Дальневосточный федеральный университет, Владивосток, Россия

${ }^{5}$ Городская клиническая больница № 15 имени О. М. Филатова, Москва, Россия

Коронавирус SARS-CoV-2, вызвавший пандемию COVID-19, проникает в клетку, связываясь с поверхностными белками: ангиотензин-превращающим ферментом 2 (АПФ2) и сериновой протеазой 2 (TMPRSS2). Экспрессия данных белков значительно различается в отдельных органах и тканях организма человека. Одним из механизмов регуляции их экспрессии является активность молекул микроРНК — коротких некодирующих РНК, важнейшей функцией которых является постранскрипционная негативная регуляция экспрессии генов. Целью работы было выявить механизмы взаимодействия изоформ микроРНК и генов АПФ2 / TMPRSS2 в тканях толстого кишечника, известных высоким уровнем экспрессии указанных ферментов. Поиск взаимодействий был осуществлен средствами корреляционного анализа на публично доступной выборке данных парного мРНК / микроРНК-секвенирования тканей кишечника. В числе находок оказались такие микроPHK, как miR-30с и miR-200c, известные своей ролью в патогенезе коронавирусной инфекции и острого респираторного дистресс-синдрома. Таким образом, были установлены новые потенциальные механизмы регуляции ферментов АПФ2 и TMPRSS2 и их возможная функциональная активность в клетке, инфицированной коронавирусом.

Ключевые слова: COVID-19, SARS-CoV-2, AПФ2, TMPRSS2, микроРHК, изосорма микроРНК, острый респираторный дистресс-синдром, коронавирус Финансирование: работа выполнена при поддержке гранта Министерства образования и науки Российской Федерации (проект RFMEFI61618X0092).

Вклад авторов: С. А. Нерсисян, М. Ю. Шкурников, А. И. Осипьянц, В. И. Вечорко - разработка концепции; С. А. Нерсисян, М. Ю. Шкурников биоинформатический анализ; С. А. Нерсисян, М. Ю. Шкурников, А. И. Осипьянц, В. И. Вечорко - интерпретация результатов; С. А. Нерсисян написание статьи.

$\triangle$ Для корреспонденции: Степан Ашотович Нерсисян ул. Вавилова, д. 7, г. Москва, 117312; s.a.nersisyan@gmail.com

Статья получена: 17.04.2020 Статья принята к печати: 28.04.2020 Опубликована онлайн: 29.04.2020

DOI: $10.24075 /$ vrgmu.2020.024

\section{ROLE OF ACE2/TMPRSS2 GENES REGULATION BY INTESTINAL microRNA ISOFORMS IN THE COVID-19 PATHOGENESIS}

Nersisyan $\mathrm{SA}^{1,2}$, Shkurnikov MYu${ }^{3}$, Osipyants $\mathrm{Al}^{3,4}$, Vechorko $\mathrm{VI}^{5}$

${ }^{1}$ National Research University Higher School of Economics, Moscow, Russia

${ }^{2}$ Lomonosov Moscow State University, Moscow, Russia

${ }^{3}$ P. A. Hertsen Moscow Oncology Research Center, branch of the National Medical Research Radiology Center, Moscow, Russia

${ }^{4}$ Far Eastern Federal University, Vladivostok, Russia

${ }^{5}$ City Clinical Hospital № 15 named after O. M. Filatov, Moscow, Russia

Coronavirus SARS-CoV-2, the cause of the COVID-19 pandemic, enters the cell by binding the cell surface proteins: angiotensin-converting enzyme 2 (ACE2) and transmembrane serine protease 2 (TMPRSS2). The expression of these proteins varies significantly in individual organs and tissues of the human body. One of the proteins' expression regulation mechanisms is based on the activity of the microRNA (miRNA) molecules, small non-coding RNAs, the most important function of which is the post-transcriptional negative regulation of gene expression. The study was aimed to investigate the mechanisms of the interactions between miRNA isoforms and ACE2/TMPRSS2 genes in the colon tissues known for the high level of expression of the described enzymes. The search for interactions was performed using the correlation analysis applied to the publicly available paired mRNA/miRNA sequencing data of colon tissues. Among the others, such miRNAs as miR-30c and miR-200c were identified known for their involvement in the coronavirus infection and acute respiratory distress syndrome pathogenesis. Thus, new potential mechanisms for the ACE2 and TMPRSS2 enzymes regulation were ascertained, as well as their possible functional activity in a cell infected with coronavirus.

Keywords: COVID-19, SARS-CoV-2, ACE2, TMPRSS2, miRNA, isomiR, acute respiratory distress syndrome, coronavirus

Funding: the study was supported by the Ministry of Science and Higher Education of the Russian Federation grant (project RFMEFI61618X0092).

Author contribution: Nersisyan SA, Shkurnikov MYu, Osipyants Al, Vechorko VI — study concept; Nersisyan SA, Shkurnikov MYu — bioinformatics analysis; Nersisyan SA, Shkurnikov MYu, Osipyants Al, Vechorko VI — interpretation of results; Nersisyan SA — manuscript writing.

$\triangle$ Correspondence should be addressed: Stepan A. Nersisyan

Vavilova, 7, Moscow, 117312; s.a.nersisyan@gmail.com

Received: 17.04.2020 Accepted: 28.04.2020 Published online: 29.04.2020

DOI: $10.24075 /$ brsmu.2020.024

Быстрое и прогрессирующее распространение коронавирусной инфекции COVID-19, вызванной коронавирусом SARS-CoV-2, глубоко повлияло на здоровье сотен тысяч людей, создав серьезный вызов системам здравоохранения и глобальной экономической стабильности. Характеристики SARS-CoV-2, особенно отличающие это заболевание от гриппа, заключаются в более высокой скорости инфицирования в сочетании с 
повышенным риском тяжелого течения и летальности, главным образом из-за возникновения острого респираторного дистресс-синдрома (ОРДС) [1]. Механизм инфицирования клетки активно изучают во многих лабораториях. В частности, уже известно, что вирусная оболочка SARS-CoV-2 экспрессирует белок SPIKE (белок S), который содержит рецепторсвязывающий домен, взаимодействующий с внеклеточным доменом ангиотензин-превращающего фермента 2 (АПФ2) с высокой аффинностью. Дальнейшее расщепление белка S сериновой протеазой 2 (TMPRSS2) для генерирования субъединиц S1 и S2 является критической стадией для слияния мембран и вирусной интернализации путем эндоцитоза с АПФ2 в легочном эпителии. Предполагается, что большую вирулентность SARS-CoV-2 по сравнению с другими коронавирусами можно объяснить тем фактом, что взаимодействие белка S1 с АПФ2 имеет заметно более сильную аффинность. Такой механизм попадания SARSCoV-2 приводит к потере АПФ2 на поверхности клетки, тем самым способствуя хронической потере легочной функции и усилению фиброза тканей [2].

МикроРНК - это короткие некодирующие одноцепочечные РНК, состоящие в среднем из 22 нуклеотидов. Одна из важнейших внутриклеточных функций микроРНК - негативная регуляция экспрессии генов, осуществляемая путем связывания молекулы микроРНК с МРНК-мишенью по принципу комплементарности, что приводит к деградации МРНК или блокировке ее трансляции [3]. МикроРНК образуются из более длинных молекул-шпилек пре-микроРНК в результате обработки ферментами Drosha и Dicer, осуществляющими разрезание шпильки [4]. Неточность в позиции разреза приводит к появлению изоформ микроРНК, различающихся несколькими нуклеотидами на концах молекулы. Показано, что для многих микроРНК их каноническая форма экспрессирована значительно слабее, чем некоторая альтернативная изоформа [5]. Крайне важно, что у различных изоформ одной микроРНК могут быть совершенно разные гены-мишени. Это объясняется тем, что наиболее важную роль при связывании с мРНК-мишенью играет участок микроРНК co 2 по 7 нуклеотид (seed-регион) на 5'-конце молекулы [6].

Известно, что функциональные нарушения микроРНК и их изоформ связаны с большим количеством патологических состояний, в том числе с онкологическими, неврологическими и сердечно-сосудистыми заболеваниями [7]. Большое число работ посвящено исследованию роли микроРНК в патогенезе вирусных инфекций: одни из них направлены на изучение возможности прямого терапевтического микроРНК-воздействия на вирус [8], тогда как другие нацелены на изучение потенциальных взаимодействий микроРНК и белков, играющих ключевую роль в процессах жизнедеятельности вируса [9]. Однако вопрос о микроРНК-регуляции экспрессии АПФ2 и TMPRSS2 при COVID-19 остается малоизученным. Целью работы было выявить механизмы взаимодействия изоформ микроРНК и генов АПФ2 / TMPRSS2 в тканях толстого кишечника, известных высоким уровнем экспрессии указанных ферментов.

\section{МАТЕРИАЛЫ И МЕТОДЫ}

Для поиска изоформ микроРНК, взаимодействующих с ферментами АПФ2 И TMPRSS2, проводили интегрированный анализ парного профиля экспрессии
МРНК и микроРНК в выборке нормальных тканей толстого кишечника, в котором данный фермент экспрессирован наиболее сильно. На выбор данной ткани повлиял также тот факт, что модели кишечника зачастую используют для исследований вирусов in vitro $[10,11]$. Так, была использована публично доступная выборка, полученная в рамках проекта The Cancer Genome Atlas Colon Adenocarcinoma (TCGA-COAD), проанализированная методами МРНК- и микроРНК-секвенирования нового поколения [12]. Данные представляют из себя матрицы экспрессии тысяч мРНК и изоформ микроРНК в восьми образцах, единица экспрессии - двоичный логарифм количества ридов, попавших на соответствующий транскрипт, нормированный на верхнюю квартиль всего распределения (FPKM-UQ). Для поиска потенциальных регуляторных взаимодействий изоформ микроРHK и TMPRSS2 рассчитывали коэффициенты корреляции Спирмена между экспрессиями 25\% самых высокоэкспрессированных изоформ с экспрессиями соответствующей мРНК, с последующей фильтрацией по p-value на уровне значимости 0,05.

\section{РЕЗУЛЬТАТЫ ИССЛЕДОВАНИЯ}

Экспрессия АПФ2 и TMPRSS2 на уровне мPHK оказалась очень большой: TMPRSS2 попал в список 1\% наиболее экспрессированных генов, тогда как экспрессия АПФ2 расположилась между 93-м и 94-м процентилями, что полностью согласуется с литературными данными [13] (см. рис.). Применение корреляционного анализа позволило выявить микроРНK miR-21, значимо отрицательно коррелирующую с экспрессией гена АПФ2, и следующие семейства микроРНК, регулирующие TMPRSS2: let-7a / let-7d, miR-30a, miR-30c, miR-127, miR194, miR-200c, miR-361 и miR-423. При этом микроРНК let-7a была представлена изоформой hsa-let-7a-5p, отличающейся от канонической добавлением аденина к 5'-концу молекулы, a miR-194 была представлена изоформой hsa-miR-194-3p, у которой отсутствует первый нуклеотид с 5'-конца. Отсутствие в этом списке соответствующих канонических форм микроРНК подчеркивает важность рассмотрения профиля всех изоформ микроРНК, а не только канонических.

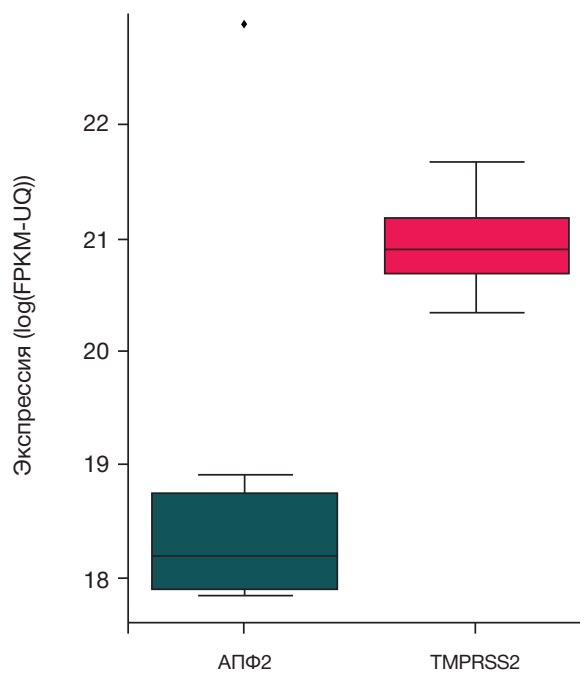

Рис. Распределение экспрессии АПФ2 и TPMRSS2 в тканях кишечника. Границы ящиков соответствуют нижнему и верхнему квартилям, вертикальный отрезок внутри ящика отображает значение медианы. Черным ромбом показан выброс 


\section{ОБСУЖДЕНИЕ РЕЗУЛЬТАТОВ}

Некоторые из найденных микроРНК уже были обнаружены в вирусологических исследованиях. Так, было показано, что экспрессия miR-30с в легких мыши значимо меняется при заражении вирусом SARS-CoV [14], что дает основание выдвинуть гипотезу о задействовании данной микроРНК в развитии болезни, вызванной вирусом. Огромный интерес представляет также микроPHK miR-200c. В 2017 г. была опубликована работа, показавшая, что эта микроРНК играет ключевую роль в патогенезе ОРДС вирусной этиологии [15]. Авторы выявили, что вирус птичьего гриппа H5N1 промотирует экспрессию miR-200с, мишенью которой служит рецептор АПФ2. Более того, были найдены вирусные белки, непосредственно отвечающие за повышение экспрессии микроРНК. Обнаружение возможности взаимодействия данной микроРНК с ферментом TMPRSS2 подчеркивает необходимость исследований по изучению роли miR-200с в патогенезе COVID-19.

\section{ВЫВОДЫ}

Полученные результаты свидетельствуют о наличии множества регуляторных взаимодействий между изосоормами микроРНК и ферментами АПФ2 / TMPRSS2. Такая информация крайне актуальна ввиду ключевой функции ферментов в механике инфицирования клетки коронавирусом SARS-CoV-2. Необходимы дальнейшие исследования для уточнения и экспериментальной валидации находок. В частности, возможно открытие новых направлений в терапии, основанных на контроле экспрессии АПФ2 и TMPRSS2 через микроРНК.

\section{Литература}

1. Munster VJ, Koopmans M, van Doremalen N, van Riel D, de Wit E. A Novel Coronavirus Emerging in China - Key Questions for Impact Assessment. N Engl J Med. 2020 Feb 20; 382 (8): 692-4.

2. Hoffmann $M$, Kleine-Weber $\mathrm{H}$, Schroeder $\mathrm{S}$, Krüger N, Herrler T, Erichsen S, et al. SARS-CoV-2 Cell Entry Depends on ACE2 and TMPRSS2 and Is Blocked by a Clinically Proven Protease Inhibitor. Cell. 2020 Mar 4. pii: S0092-8674(20)30229-4.

3. Nilsen TW. Mechanisms of microRNA-mediated gene regulation in animal cells. Trends Genet. 2007 May; 23 (5): 243-9.

4. Makarova JA, Shkurnikov MU, Turchinovich AA, Tonevitsky AG, Grigoriev Al. Circulating microRNAs. Biochemistry (Mosc). 2015 Sep; 80 (9): 1117-26.

5. Loher P, Londin ER, Rigoutsos I. IsomiR expression profiles in human lymphoblastoid cell lines exhibit population and gender dependencies. Oncotarget. 2014 Sep 30; 5 (18): 8790-802.

6. Mazière $P$, Enright AJ. Prediction of microRNA targets. Drug Discov Today. 2007 Jun; 12 (11-12): 452-8.

7. Osip'yants Al, Knyazev EN, Galatenko AV, Nyushko KM, Galatenko W, Shkurnikov MY, et. al. Changes in the Level of Circulating hsamiR-297 and hsa-miR-19b-3p miRNA Are Associated with Generalization of Prostate Cancer. Bull Exp Biol Med. 2017 Jan; 162 (3): 379-82.

8. Leon-Icaza SA, Zeng M, Rosas-Taraco AG. microRNAs in viral acute respiratory infections: immune regulation, biomarkers,

\section{References}

1. Munster VJ, Koopmans M, van Doremalen N, van Riel D, de Wit E. A Novel Coronavirus Emerging in China - Key Questions for Impact Assessment. N Engl J Med. 2020 Feb 20; 382 (8): 692-4.

2. Hoffmann M, Kleine-Weber $\mathrm{H}$, Schroeder $\mathrm{S}$, Krüger N, Herrler T, Erichsen S, et al. SARS-CoV-2 Cell Entry Depends on ACE2 and TMPRSS2 and Is Blocked by a Clinically Proven Protease Inhibitor. Cell. 2020 Mar 4. pii: S0092-8674(20)30229-4.

3. Nilsen TW. Mechanisms of microRNA-mediated gene regulation in animal cells. Trends Genet. 2007 May; 23 (5): 243-9.

4. Makarova JA, Shkurnikov MU, Turchinovich AA, Tonevitsky AG, Grigoriev Al. Circulating microRNAs. Biochemistry (Mosc). 2015 Sep; 80 (9): 1117-26.

5. Loher P, Londin ER, Rigoutsos I. IsomiR expression profiles in human lymphoblastoid cell lines exhibit population and gender dependencies. Oncotarget. 2014 Sep 30; 5 (18): 8790-802.

6. Mazière $P$, Enright AJ. Prediction of microRNA targets. Drug Discov Today. 2007 Jun; 12 (11-12): 452-8.

7. Osip'yants Al, Knyazev EN, Galatenko AV, Nyushko KM, Galatenko W, Shkurnikov MY, et. al. Changes in the Level of Circulating hsamiR-297 and hsa-miR-19b-3p miRNA Are Associated with Generalization of Prostate Cancer. Bull Exp Biol Med. 2017 Jan; 162 (3): 379-82.

8. Leon-Icaza SA, Zeng M, Rosas-Taraco AG. microRNAs in viral acute respiratory infections: immune regulation, biomarkers, therapy, and vaccines. ExRNA. 2019 Feb; 1.

9. Mallick B, Ghosh Z, Chakrabarti J. MicroRNome analysis unravels the molecular basis of SARS infection in bronchoalveolar stem cells. PLoS One. 2009 Nov 13; 4 (11): e7837.

10. Samatov TR, Senyavina NV, Galatenko VV, Trushkin EV, Tonevitskaya SA, Alexandrov DE, et al. Tumour-like druggable gene expression pattern of $\mathrm{CaCo} 2$ cells in microfluidic chip. BioChip J. 2016 Jul; 10: 215-20.

11. Sakharov D, Maltseva D, Knyazev E, Nikulin S, Poloznikov A, Shilin S, et. al. Towards embedding Caco-2 model of gut interface in a microfluidic device to enable multi-organ models for systems 
biology. BMC Syst Biol. 2019 Mar 5; 13 (Suppl 1): 19.

12. Cancer Genome Atlas Network. Comprehensive molecular characterization of human colon and rectal cancer. Nature. 2012 Jul 18; 487 (7407): 330-7.

13. Vaarala MH, Porvari KS, Kellokumpu S, Kyllönen AP, Vihko PT. Expression of transmembrane serine protease TMPRSS2 in mouse and human tissues. J Pathol. 2001 Jan; 193 (1): 134-40.
14. Peng X, Gralinski L, Ferris MT, Frieman MB, Thomas MJ, Proll S, et. al. Integrative deep sequencing of the mouse lung transcriptome reveals differential expression of diverse classes of small RNAs in response to respiratory virus infection. mBio. 2011 Nov 15; 2 (6).

15. Liu Q, Du J, Yu X, Xu J, Huang F, Li X, Zhang C, Li X, et al. miRNA200c-3p is crucial in acute respiratory distress syndrome. Cell Discov. 2017 Jun 27; 3: 17021. 